\title{
RoHS-konforme, thermoelektrisch gekühlte Detektoren für das langwellige Infrarot auf Basis antimonidischer Übergitter
}

\author{
Raphael Müller, Jasmin Niemasz, Volker Daumer, Robert Rehm \\ Fraunhofer Institut für Angewandte Festkörperphysik (IAF) \\ Tullastraße 72, 79108 Freiburg, Deutschland
}

\begin{abstract}
Zusammenfassung
In diesem Beitrag präsentieren wir antimonidische Übergitter, im Speziellen InAs/GaSb-Übergitter, als ein zu Quecksilbercadmiumtellurid alternatives Materialsystem für die schnelle und sensitive Photodetektion im langwelligen Infrarot bei Betriebstemperaturen die mit thermoelektrischer Kühlung erreichbar sind. Die Detektorentwicklung für InAs/GaSb-Übergitter, welche unter anderem durch die mangelnde RoHS-Konformität von Detektoren aus Quecksilbercadmiumtellurid motiviert wird, wird für das Detektionskonzept eines lateral betriebenen Photoleiters vorgestellt. Die in diesem Beitrag diskutierten Detektoren zeigen eine Grenzwellenlänge von 10,5 $\mu \mathrm{m}$ bei einer Betriebstemperatur von $210 \mathrm{~K}$, erreichbar mithilfe eines 3-stufigen Peltierkühlers. Die durchschnittliche Detektivität der undotierten Übergitterdetektoren ist vergleichbar mit derer von dotieroptimierten Detektoren auf Basis von Quecksilbercadmiumtellurid. Die experimentelle Untersuchung der Übergitterdotierung stellt eine Erhöhung der Detektivität und eine zunehmende Konkurrenzfähigkeit mit Quecksilbercadmiumtellurid für Detektoranwendungen im langwelligen Infrarot unter thermoelektrischer Kühlung in Aussicht.
\end{abstract}

Keywords: Infrarotdetektor, Peltierkühler, RoHS, InAs/GaSb-Übergitter, MCT

\section{Thermoelektrisch gekühlte Infrarot- Photodetektoren}

Für die Identifizierung von Substanzen, die Bestimmung ihrer Konzentration in Gasen und Flüssigkeiten sowie die Messung zeitlich veränderlicher Signale werden in Forschung und Industrie schnelle und sensitive Infrarotdetektoren (IR-Detektoren) benötigt und standardmäßig eingesetzt.

Die Kombination aus schneller Signaldetektion und hohem Signal-Rausch-Verhältnis, welches die aussagekräftige Detektion selbst schwacher Signale ermöglicht, erfordert den Einsatz von IR-Photodetektoren. Diese basieren auf Halbleitermaterialien und werden für verbesserte Leistungsfähigkeit zumeist gekühlt betrieben. Die thermoelektrische Kühlung, welche auf dem Einsatz mehrstufiger Peltierkühler beruht, ermöglicht Betriebstemperaturen zwischen Raumtemperatur und etwa $200 \mathrm{~K}$ und ist für viele Anwendungsszenarien das einzige praktikable und kosteneffiziente Kühlverfahren für IR-Detektoren.

In Abb. 1 ist ein IR-Detektor mit und ohne Gehäuse auf einem 4-stufigen Peltierkühler exemplarisch dargestellt. Oberhalb des Peltierkühlers befindet sich eine Mikrolinse, welche die einfallende Strahlung auf das darunterliegende Detektorelement fokussiert und dadurch dessen Signal-Rausch-Verhältnis steigert.

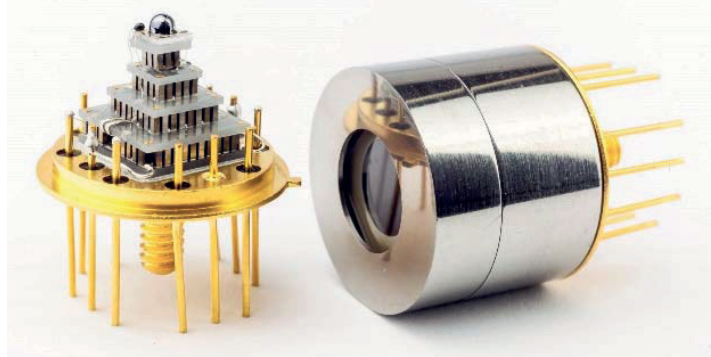

Abb. 1: IR-Detektor mit Immersionslinse auf 4-stufigem Peltierkühler [1]

Thermoelektrisch gekühlte Detektoren auf Basis von Quecksilbercadmiumtellurid (MCT, von engl.: mercury cadmium telluride) sind in verschiedenen Größen, mit unterschiedlichen Grenzwellenlängen und für den durch thermoelektrische Kühlung erschließbaren Temperaturbereich kommerziell erhältlich. Die Nutzung von Quecksilber und Cadmium für die Herstellung von IR-Detektoren wird jedoch zunehmend durch die RoHS-Richtlinie »2011/65/EU« der europäischen Union bedroht, welche deren Nutzung beschränkt. Derzeit existieren für bestimmte Anwendungen befristete Ausnahmegenehmigungen, für die mittel- und langfristige Detektorentwicklung werden Alternativen jedoch intensiv beforscht. 
Flexible Materialsysteme für die Infrarotdetektion: Quecksilbercadmiumtellurid und InAs/GaSb-Übergitter

Die zentrale Eigenschaft für die Eignung eines Halbleiters für die Herstellung von IRPhotodetektoren ist dessen Bandlücke $E_{g}$, welche maßgeblich die Grenzwellenlänge des Detektors bestimmt.

Die Bandlücke von MCT, respektive die Grenzwellenlänge von MCT-Detektoren, kann mithilfe verschiedener Epitaxieverfahren durch das Verhältnis von Cadmium und Quecksilber eingestellt werden. Dabei sind Grenzwellenlängen von 2-30 $\mu \mathrm{m}$ möglich, wobei die Einstellbarkeit der Bandlücke sowie flächig homogenes Wachstum für die höheren Grenzwellenlängen in diesem Bereich jedoch technologisch zunehmend komplizierter wird.

Das Legieren von III/V-Halbleitern wie In, Ga, As, $\mathrm{Sb}$ ermöglicht ebenfalls eine flexible Bandlückeneinstellbarkeit, jedoch in einem schmaleren Spektralbereich und mit geringerer maximal erreichbarer Grenzwellenlänge.

Eine größere Flexibilität besteht durch den Einsatz sogenannter Kompositionsübergitter, einer periodischen Abfolge alternierender Schichten verschiedener Materialien, die im vorgestellten Fall der InAs/GaSb Übergitter aus Indiumarsenid und Galliumantimonid bestehen. Diese Schichten können durch Molekularstrahlepitaxie mit Schichtdicken im Bereich weniger Monolagen (ML) und abrupten Schichtübergängen hergestellt werden. In diesem künstlichen Halbleitermaterial werden die energetische Lage von Leitungs- und Valenzbandkante durch die Quantenmechanik definiert. Durch die Superposition der Wellenfunktionen der Ladungsträger in den Übergitterteilschichten entstehen sogenannte Minibänder. Die energetische Lage und der Abstand dieser Minibänder definiert die Größe der effektiven Halbleiterbandlücke. Durch Veränderung der Teilschichtdicken von InAs und GaSb in einer Übergitterperiode können die energetische Lage der Minibänder und die Größe der Bandlücke zielgenau eingestellt werden.

In Abb. 2 ist die Bandanordnung von InAs und GaSb sowie das resultierende Bandstrukturdiagramm eines InAs/GaSb-Übergitters dargestellt. In der Bandstruktur des Übergitters sind das energetisch tiefstliegende Elektronenminiband (Übergitterleitungsband) und das energetisch höchstliegende Lochminiband (Übergittervalenzband) exemplarisch dargestellt. Eine Verbreiterung der InAs-Teilschichtdicke führt beispielweise zur Absenkung des Übergitterleitungsbandes und zur Verringerung der Übergitterbandlücke.

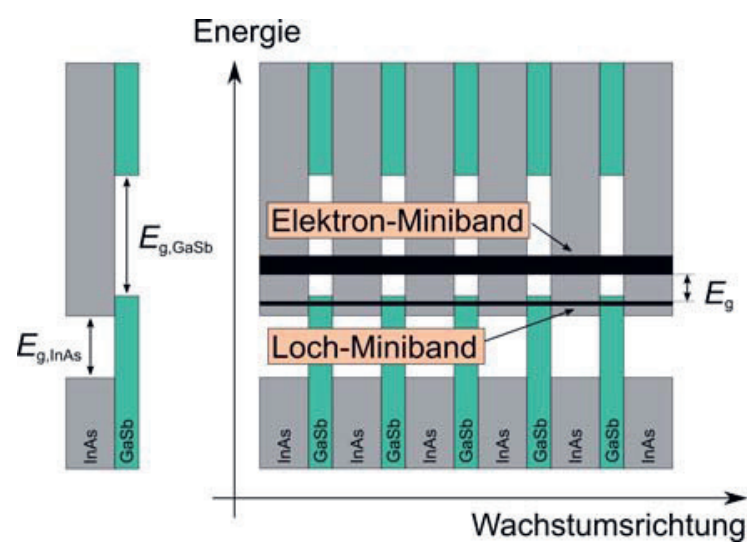

Abb. 2: Bandanordnung von InAs und GaSb sowie Bandstrukturdiagramm eines InAs/GaSb-Übergitters

Trotz der theoretisch aufwändigen Beschreibung lassen sich InAs/GaSb-Übergitter für viele entwicklungs- und anwendungsspezifische Aspekte als anisotropes Halbleitermaterialsystem mit flexibel einstellbarer Bandlücke betrachten mit dem Detektorgrenzwellenlängen im Infraroten für verschiedene Anwendungen im Bereich von 3-20 $\mu \mathrm{m}$ maßgeschneidert definiert werden können.

Im Vergleich zu MCT zeichnen sich Übergitter durch höhere Prozessausbeuten und geringere Kosten aus. Aus diesen wirtschaftlichen Gründen und aufgrund einer theoretisch postuliert, höheren Leistungsfähigkeit als MCT werden InAs/GaSb-Übergitter seit einigen Jahren als Alternative zu MCT intensiv beforscht und zunehmend konkurrenzfähig. Der Fokus der Übergitterentwicklung liegt auf bildgebenden Anwendungen bei denen das Detektormaterial kryogen gekühlt betrieben wird. Auch das Fraunhofer IAF erforscht und fertigt seit langem IR-Detektoren auf Basis von InAs/GaSb-Übergittern, was zur Entwicklung von bispektralen Kameras für das mittelwellige Infrarot [2] und zur ersten europäischen InAs/GaSb-Übergitterkamera für das langwellige Infrarot führte [3].

\section{Entwicklung und Herstellung photoleitender Detektoren}

Die hier vorgestellte Entwicklung photoleitender Detektoren erfolgte in Kooperation mit VIGO System, im Folgenden kurz "VIGO», für MIRPHAB. MIRPHAB ist eine europäische Pilotlinie für die Entwicklung und Kommerzialisierung von sogenannten "Advanced MIRPHAB Modules", bestehend aus Lichtquelle, Lichtleiter und Detektor. Diese Module werden für den Spektralbereich von 3-12 $\mu \mathrm{m}$ entwickelt und finden Anwendung in der chemischen Analyse und der Spektroskopie (siehe auch: www.mirphab.eu). 
Der Schichtentwurf und das Wachstum der Übergitterschichten erfolgen am Fraunhofer IAF, ebenso wie die photolithographische Prozessierung. Die im Folgenden knapp vorgestellte Schichtstruktur, das Detektorkonzept, sowie die Prozessierung wurden bereits in Ref. [4] und Ref. [5] diskutiert.

In Abb. 3 sind die Schichtstruktur sowie ein prozessiertes Detektorelement schematisch dargestellt. Für die in Abb. 3 oben gezeigte Schichtstruktur wird auf 3"-GaAs zunächst ein geeigneter Wachstumspuffer abgeschieden, gefolgt vom photosensitiven Übergitterabsorber und einer Kontaktschicht. Für die Übergitterkomposition der Absorberschicht, welche die Grenzwellenlänge des Detektors definiert, wurden $14 \mathrm{ML}$ InAs und $7 \mathrm{ML}$ GaSb gewählt. Im prozessierten Detektorelement, in Abb. 3 unten, sind neben den strukturierten Schichten von Absorber und Kontakt eine Passivierungsschicht sowie die Metallisierung der Kontakte gezeigt. Um für ein durch das Substrat beleuchteten Detektor einen Doppeldurchgang der einfallenden Strahlung zu ermöglichen, wird zudem eine als Spiegel wirkende Metallisierung im mittigen Bereich des photosensitiven $\mathrm{Ab}$ sorbers zwischen den metallischen Kontakten aufgebracht.

\begin{tabular}{|lr|}
\hline Übergitter-Kontaktschicht & $1 \mu \mathrm{m}$ \\
\hline $\begin{array}{l}\text { Übergitter-Absorber } \\
\text { (14ML InAs/7ML GaSb) }\end{array}$ & $5 \mu \mathrm{m}$ \\
\hline Puffer & $2 \mu \mathrm{m}$ \\
\hline $\begin{array}{l}\text { Substrat } \\
\text { (semiisolierendes GaAs) }\end{array}$ & $1100 \mu \mathrm{m}$ \\
\hline
\end{tabular}

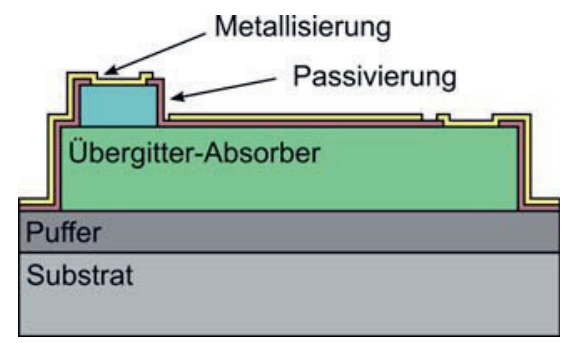

Abb. 3: Schichtstruktur für die Herstellung von Übergitterdetektoren (oben) und

Schema eines prozessierten

Übergitterdetektors (unten)

Für die Vereinzelung der Detektorstrukturen und die weitere Integration werden VIGO Waferchips zur Verfügung gestellt. Einer dieser Waferchips, welcher $15 \times 15$ Detektorelemente umfasst, ist in Abb. 4 dargestellt. Nach der Detektorvereinzelung werden Mikrolinsen aus dem GaAs-Substrat abgeformt, die Detektoren mit Verstärkerelektronik versehen und in einem dedizierten Messaufbau charakterisiert.

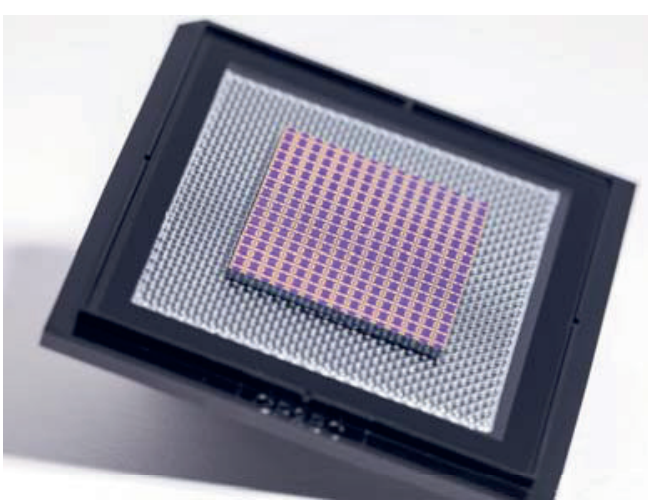

Abb. 4: Waferchip mit 15x15 InAs/GaSbÜbergitterphotoleitern $\left(22 \times 22 \mathrm{~mm}^{2}\right)$

\section{Spektrale Detektivität: \\ Zentrale Vergleichsgröße für die Bewertung von Infrarotdetektoren}

Die zentrale Vergleichsgröße für die Bewertung der Leistungsfähigkeit von Infrarotdetektoren ist die spektrale Detektivität $D^{*}(\lambda)$, ein Maß für das Signal-Rausch-Verhältnis des Detektors. Sie verknüpft das durch die eingestrahlte Leistung normierte Photosignal, die Responsivität $R$, mit dem Rauschstrom $I_{n}$, normiert mit der optischen Detektorfläche $A$ und der Bandbreite $\Delta f$ :

$$
D^{*}(\lambda)=\frac{R(\lambda)}{I_{n}(f)} \sqrt{A \Delta f} .
$$

Die Bestimmung der Detektivität erfordert folglich neben der Kenntnis der Detektorgeometrie die Bestimmung der spektralen Responsivität sowie des frequenzabhängigen Rauschverhaltens.

Für den Fall des hier untersuchten Detektorkonzepts eines photoleitenden InAs/GaSbÜbergitterdetektors, welcher unter thermoelektrischer Kühlung betrieben wird, ist die Responsivität proportional zur Quanteneffizienz und dem Photoleitungsgewinn, das heißt der Lebensdauer der Ladungsträger sowie deren Beweglichkeit. Der frequenzabhängige Rauschstrom wird als Kombination aus thermischem Rauschen, Generations-Rekombinationsrauschen sowie 1/f-Rauschen beschrieben, welche vom Widerstand des Detektors, dem Photoleitungsgewinn sowie unter anderem der Detektorprozessierung abhängig sind.

Eine Verbesserung der Detektivität kann aus einer Erhöhung der materialspezifischen, dotierabhängigen Ladungsträgerlebensdauer oder Beweglichkeit, sowie durch die Erhöhung des Detektorwiderstands resultieren.

Nachfolgend werden nun zunächst Charakterisierungsergebnisse undotierter Übergitterphotoleiter diskutiert und dann Ergebnisse einer Dotierstudie vorgestellt. 


\section{Spektrale Detektivität undotierter InAs/GaSb-Übergitterphotoleiter}

Für die elektrooptische Bewertung der vorgestellten Detektoren ist neben der potenziellen Leistungsfähigkeit auch die Homogenität über den Wafer von großer Bedeutung für eine mögliche Kommerzialisierbarkeit.

Für eine erste Bewertung der Materialhomogenität wurden 10 zufällig ausgewählte Detektoren mit einer Detektorfläche von $100 \times 100 \mu \mathrm{m}^{2}$ aufgebaut. Auf den detektivitätssteigernden Einfluss einer GaAs-Immersionslinse wurde hier verzichtet, die Kompatibilität wurde bereits in Ref. [5] gezeigt. Nach dem Aufbau der Detektoren mit planer Substratoberfläche erfolgte die Charakterisierung der Responsivität und des Rauschverhaltens bei einer Betriebstemperatur von $210 \mathrm{~K}$, welche mit einem 3-stufigen Peltierkühler erreichbar ist.

Die Charakterisierung der einzelnen Detektoren wurde bereits in Ref. [4] diskutiert. Dabei konnte ein sehr einheitliches Verhalten festgestellt werden, sowohl für die Responsivität, wobei die Streuung der Maximalwerte im Bereich der messplatzbedingten Unsicherheit lag, als auch für das Rauschverhalten, welches sowohl qualitativ als auch quantitativ sehr einheitlich war. Demzufolge ergab sich ebenfalls ein einheitliches Verhalten bezüglich der Detektivität der untersuchten Übergitterphotoleiter. Dies motiviert für die weitere Diskussion den Mittelwert der Detektivität dieser 10 Detektoren als repräsentativen Wert zu nutzen.

In Abb. 5 ist die gemittelte spektrale Detektivität der 10 InAs/GaSb-Übergitterphotoleiter vorgestellt. Außerdem ist die Detektivität für kommerziell erhältliche MCT-Photoleiter von VIGO mit unterschiedlicher Grenzwellenlänge gezeigt, welche den direkten Vergleich ermöglichen. Die Messdaten aller Detektoren, von MCT und Übergitter, wurden am gleichen Messaufbau bei gleicher Betriebstemperatur charakterisiert und sind für das Rauschen des Detektors bei $20 \mathrm{kHz}$ angegeben. Die gemittelte Detektivität der Übergitterphotoleiter mit einer Grenzwellenlänge von $10,5 \mu \mathrm{m}$ liegt bei $3,3 \times 10^{8} \mathrm{cmHz}^{0.5} \mathrm{~W}^{-1}$ im Maximum und $1,6 \times 10^{8} \mathrm{cmHz}^{0.5} \mathrm{~W}^{-1}$ bei der typischen Grenzwellenlänge von 10,6 $\mu \mathrm{m}$. Der Vergleich mit der Kurve entsprechender Grenzwellenlänge bei MCT (blau) offenbart geringere Werte für die Übergitterdetektoren, der Maximalwert für MCT ist dabei jedoch weniger als zweimal so hoch.

Trotz der etwas höheren Detektivität von dotieroptimiertem MCT im Vergleich zu undotierten Übergittern, zeigt dies die Relevanz des Übergittermaterialsystems für den Einsatz unter Betriebstemperaturen die mit Peltierkühlern erreichbar sind.



Abb. 5: Gemittelte spektrale Detektivität von 10 undotierten InAs/GaSb-Übergitterphotoleitern im Vergleich zu dotiertem kommerziellem MCT mit Grenzwellenlänge $\lambda_{g}$ von 9 bis $13 \mu \mathrm{m}$

\section{Entwicklung und Bewertung dotierter InAs/GaSb-Übergitterphotoleiter}

Die Responsivität und der Dunkelwiderstand sind wichtige, die Detektivität des Photoleiters beeinflussende Eigenschaften, welche maßgeblich durch die Dotierung der photosensitiven Absorberschicht beeinflusst werden. Dies motiviert die Untersuchung der Übergitterdotierung im Rahmen der Detektoroptimierung. Wir zeigen im Folgenden erste Ergebnisse einer Dotierstudie für InAs/GaSb-Übergitterphotoleiter mit gleicher Übergitterkomposition von $14 \mathrm{ML}$ InAs und $7 \mathrm{ML}$ GaSb bei variierter Dotierung der Absorberschicht, ebenfalls basierend auf GaAs-Substrat. Die Dotierung der Absorberschicht erfolgt durch den Einbau von Beryllium in die InAs-Teilschicht, was zunächst zur Kompensation der n-artigen Hintergrunddotierung und mit steigender Dotierkonzentration zunehmend zu p-leitenden Eigenschaften der Schicht führt.

Die vorgestellten Messdaten wurden für jeweils einen Detektor der jeweiligen Dotierniveaus in einem Tauschgaskryostaten. Die untersuchten Detektorstrukturen haben eine optisch sensitive Fläche von $1 \times 1 \mathrm{~mm}^{2}$, keine Frontseitenmetallisierung und wurden unter Frontseitenbeleuchtung charakterisiert. Für alle Detektorstrukuren werden der Dunkelwiderstand und der Photostrom gemessen, letzterer mithilfe einer Lockin-Messtechnik bei frequenzmodulierter Beleuchtung mit einem Schwarzkörperstrahler der Temperatur $1100^{\circ} \mathrm{C}$.

In Abb. 6 sind die Widerstände für einen undotierten Übergitterdetektor sowie drei Übergitterdetektoren mit zunehmender Be-Dotierung für eine Betriebstemperatur von $210 \mathrm{~K}$ gezeigt. Bei der angegebenen Dotierung handelt es sich um die nominelle Dotierung der InAs-Teilschicht, bezogen auf die Gesamtdicke einer Übergitterperiode. Die Messdaten zeigen 


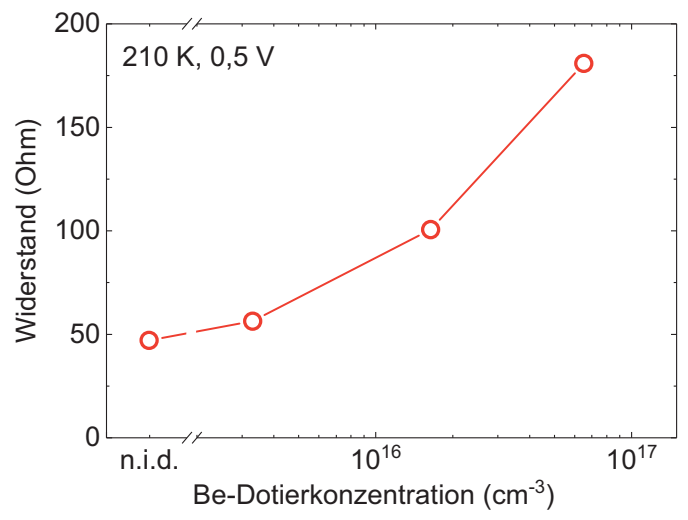

Abb. 6: Dotierabhängigkeit des Widerstands photoleitender InAs/GaSb-Übergitterdetektoren bei $210 \mathrm{~K}$ und $0,5 \mathrm{~V}$

einen eindeutigen Trend eines mit zunehmender Dotierung steigenden Widerstands mit $46 \mathrm{Ohm}$ für einen undotierten Übergitterdetektor bis zu $181 \mathrm{Ohm}$ für den mit $6,7 \times 10^{16} \mathrm{~cm}^{-3}$ höchstdotierten Übergitterdetektor dieser Untersuchung. Dies führt zu einer Reduktion des thermischen Rauschens des Detektors.

In Abb. 7 ist der Photostrom für die gleichen Detektorstrukturen bei gleicher Betriebstemperatur und Betriebsspannung dargestellt. Die vertikale Achse ist auf den Photostrom der undotierten Probe normiert. Für die untersuchten Proben zeigt sich ein mit zunehmender Dotierung zunächst steigender Photostrom, der ein um einen Faktor 2,7 erhöhtes Photostromniveau ermöglicht. Bei hoher Dotierung fällt dieses jedoch wieder nahezu auf den Wert des undotierten Übergitters ab. Die Verringerung des Photostroms für stark erhöhte Dotierpegel ist vermutlich auf eine dotierbedingte Zunahme von Rekombinations- und Streuzentren zurückzuführen, welche die Lebensdauer und die Beweglichkeit der Ladungsträger einschränken, was Responsivität und Detektivität wieder reduziert. Der generelle Trend des Photostroms deutet die Existenz eines von der Absorberdotierung abhängigen Maximalwertes an, der im Rahmen dieser Untersuchung jedoch nicht weiter quantifizierbar ist.

Zusammenfassend ist festzuhalten, dass sowohl der Detektorwiderstand als auch Photostrom durch Absorberdotierung eine Erhöhung der Detektivität in Aussicht stellen. Für die vorgestellte Betriebstemperatur von $210 \mathrm{~K}$, welche mithilfe eines dreistufigen Peltierkühlers erreichbar ist, wird diese bei der gegebenen Übergitterkomposition für eine Dotierkonzentration um $10^{16} \mathrm{~cm}^{-3}$ erwartet, bei welcher der Detektorwiderstand steigend und das Photostromniveau verglichen mit dem undotierten Fall noch stark erhöht ist.

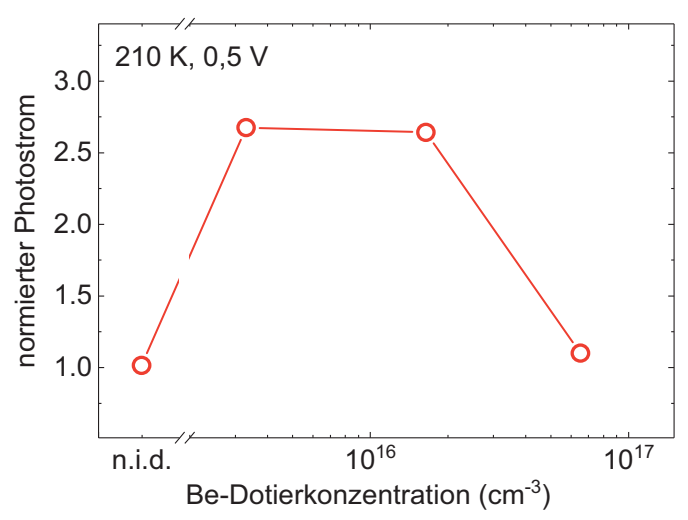

Abb. 7: Dotierabhängigkeit des Photostroms photoleitender InAs/GaSb-Übergitterdetektoren bei $210 \mathrm{~K}$ und $0,5 \mathrm{~V}$

Eine quantitative Aussage über die Erhöhung der spektralen Detektivität für dotierte Übergitterphotoleiter erfordert die Untersuchung einer größeren Anzahl von Detektoren sowie die ausstehende Charakterisierung der Detektoren im finalen Aufbauzustand unter substratseitiger Beleuchtung im Messaufbau bei VIGO. Eine vollständige Analyse wird an anderer Stelle veröffentlicht.

\section{Fazit}

In diesem Beitrag wurden InAs/GaSb-Übergitterphotodetektoren als mögliche Alternative zu Quecksilbercadmiumtellurid für mit Peltierkühlern erreichbare Betriebstemperaturen vorgestellt. Die Entwicklung eines photoleitenden Übergitterdetektors mit einer Übergitterkomposition aus 14 Monolagen InAs und 7 Monolagen $\mathrm{GaSb}$ im photosensitiven Absorber wurde vom Schichtentwurf bis zur Charakterisierung skizziert. Es wurde gezeigt, dass die spektrale Detektivität undotierter photoleitender Detektoren bei $210 \mathrm{~K}$ bereits vergleichbar ist mit der Detektivität kommerziell erhältlicher MCTDetektoren und dass durch gezielte Dotierung sowohl Widerstand als auch Photostrom erhöht werden können. Dies resultiert in einem geringeren thermischen Rauschen und einer erhöhten Responsivität, wovon beides eine Detektivitätserhöhung in Aussicht stellt. Eine Quantifizierung dieses Effekts für den finalen Aufbauzustand mit oder ohne Mikrolinse ist noch ausstehend. Nichtsdestotrotz sind InAs/GaSb-Übergitter für thermoelektrisch gekühlte IR-Detektoranwendungen bereits im vorgestellten Entwicklungsstadium als mögliche Alternative zu Quecksilbercadmiumtellurid anzusehen, dessen Nutzung in absehbarer Zeit von der RoHS-Richtlinie beschränkt wird. 


\section{Ausblick}

Die für die Entwicklung von photoleitenden InAs/GaSb-Übergitterdetektoren eingesetzten Prozesse im Bereich des Schichtwachstums und der Übergitterprozessierung am Fraunhofer IAF ermöglichen, wie in Ref. [4] gezeigt, die Herstellung einheitlich leistungsfähiger IR-Detektoren. Dies stellt hohe Prozessausbeuten und eine kosteneffiziente Nutzung der Übergittertechnologie in Aussicht.

Darüber hinaus besteht die begründete Annahme, dass die Optimierung photoleitender InAs/GaSb-Übergitterdetektoren durch BeDotierung, welche hier lediglich für die Schichtkomposition aus 14 Monolagen InAs und 7 Monolagen GaSb für $210 \mathrm{~K}$ gezeigt wurde, auch für andere Übergitterkompositionen und andere durch thermoelektrische Kühlung erreichbare Betriebstemperaturen möglich ist.

Im Kontext unterschiedlicher Übergitterkompositionen, und der damit verbundenen unterschiedlichen Detektorgrenzwellenlängen, sei an dieser Stelle nochmals auf Abb. 5 verwiesen. Sie wurde zuvor für die Einordnung der Leistungsfähigkeit undotierter Übergitterdetektoren genutzt. Der Maximalwert der Detektivität der MCT-Detektoren unterschiedlicher Grenzwellenlänge nimmt für steigende Grenzwellenlängen ab. Dieser generelle Trend ist nicht materialspezifisch, sondern auf eine allgemeine Eigenschaft von Photodetektoren zurückzuführen. Für größere Grenzwellenlängen wird eine geringe Bandlücke benötigt, was jedoch die thermische Band-BandAnregung im Halbleiter verstärkt. Diese Anregung führt $\mathrm{zu}$ hohen Ladungsträgerdichten, hohen Dunkelströmen, zunehmendem Rauschen und einer entsprechend verringerten spektralen Detektivität. Das gleiche qualitative Verhalten, die Reduktion der spektralen Detektivität für größere Grenzwellenlängen, ist daher auch bei InAs/GaSbÜbergitterphotodetektoren zu erwarten. Der quantitative Verlauf ist jedoch außerdem abhängig von fundamentalen Halbleitereigenschaften, die für Übergitterdetektoren bei hohen Betriebstemperaturen bislang noch wenig erforscht sind.

\section{Literaturnachweis}

[1] www.vigo.com.pl/products/pci-4te/

[2] R. Rehm, M. Walther, J. Schmitz, J. Fleißner, J. Ziegler, W. Cabanski, R. Breiter, Proc. of SPIE 6294, 629404 (2006); doi:10.1117/12.680495

[3] R. Rehm, V. Daumer, T. Hugger, N. Kohn, W. Luppold, R. Müller, J. Niemasz, J. Schmidt, F. Rutz, T.Stadelmann, M. Wauro, A. Wörl, Proc. of SPIE 9819, 98190X-1 (2016); doi: $10.1117 / 12.2223887$
[4] R. Müller, J. Niemasz, L. Kirste, V. Daumer, A. Janaszek, J. Jureńczyk, R. Rehm, Proc. of SPIE 10914, 1091416 (2019); doi: $10.1117 / 12.2506624$

[5] R. Müller, V. Gramich, M. Wauro, J. Niemasz, L. Kirste, V. Daumer, A. Janaszek, J. Jureńczyk, R. Rehm, Infrared Physics \& Technology 96, 141-144 (2019); doi: 10.1016/j.infrared.2018.10.019 